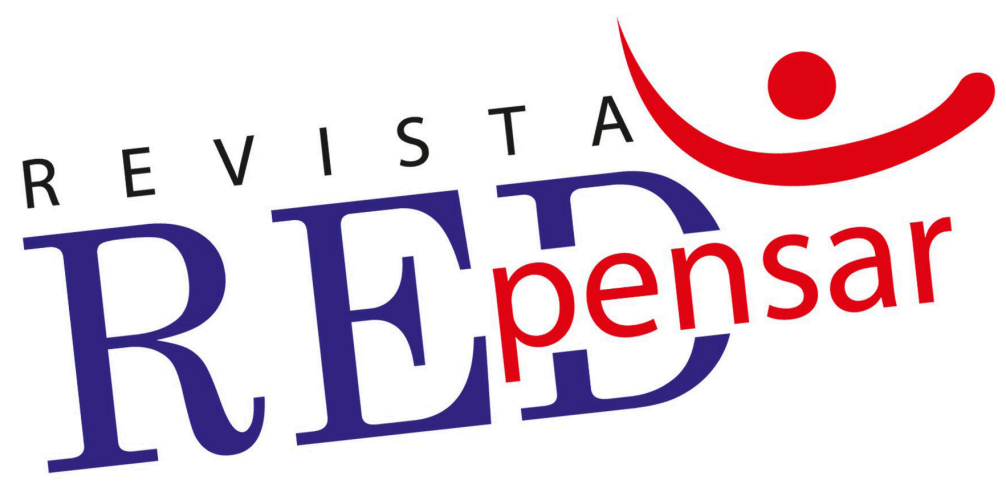

\title{
El reconocimiento como mediación vital para el cuidado
}

\section{del Otro}

The recognition as vital mediation for the care the other

\section{William Perdomo Rodriguez. Margarita Ortega Sáchica}

Revista REDpensar, volumen 5, número 2, Ago-Dic 2016

ISSNe: 2215-5384

Lanzando la RED 3 Recibido: 19 de setiembre del 2017 Aceptado: 21 de diciembre del 2017 Publicado: 07 de abril de 2018

DOI: 10.31906/redpensar.v5i2.120

\section{다(i)(2)}




\title{
El reconocimiento como mediación vital para el cuidado del Otro
}

\author{
The recognition as vital mediation for the care the other
}

William Perdomo Rodríguez. ${ }^{1}$

Margarita Ortega Sáchica ${ }^{2}$

\section{Resumen}

El artículo pretende una reflexión acerca del reconocimiento como elemento vital para el cuidado de la vida. Más allá de una simple atención y de cubrir una necesidad, el concepto-cuidado- se entiende como emergente del reconocimiento de sí mismo y del Otro, vital para el cohabitar de las personas. Lo anterior, en la premisa que para cuidar necesitamos una conexión verdadera, emocional y afectiva, que dé importancia al Otro. Así las cosas, el texto lleva hacia una reflexión de la vida misma como un soporte vital de cuidado de sí mismo y del Otro. Se realiza la introspección acerca de los términos reconocimiento y cuidado, haciendo un análisis de postulados de autores como Boff, Noddings, Gilligan, Maturana, entre otros. En esta mirada, se requiere ahondar en la perspectiva del cuidado como un modo de ser, como condición inequívoca de que el cuidado no es un acto, sino una actitud, por otra parte, el cuidado como un eje central en la formación del Ser y de la relación de las personas. La tarea de priorizar la manera en que nos relacionamos como eje central del contexto pedagógico sigue siendo un ejercicio ético urgente, posicionarnos desde un cuerpo vivo que reconoce al Otro en su diferencia, será entonces la reflexión que sin ánimos de concluir quede abierta para más diálogos y emergencias de conocimiento en esta área.

Palabras Clave: RECONOCIMIENTO, CUIDADO, ACTITUD, OTRO.

\section{Abstract}

The article aims to reflect on recognition as a vital element for the care of life. Beyond simple attention and covering a need, the concept -care- is understood as emerging from the recognition of oneself and the Other, vital for the cohabitation of people. The above, in the premise that to care we need a true and emotional connection, that gives importance to the Other. In this way, the text leads to a reflection of life itself as a vital support for caring for oneself and the Other. Introspection is done about the terms recognition and care, making an analysis of postulates of authors such as Boff, Noddings, Gilligan, Maturana, among others. In this view, it is necessary to delve into the perspective of care as a way of being, as an unambiguous condition that care is not an act, but an attitude, on the other hand, care as a central axis in the formation of the Self and of the relationship of people. The task of prioritizing the way in which we relate as the central axis of the pedagogical context remains an urgent ethical exercise, positioning ourselves from a living body that recognizes the Other in its difference, it will then be the reflection that without the intention of concluding is open for more dialogues and knowledge emergencies in this area.

Keywords: RECOGNITION, CARE, ATTITUDE, OTHER.

1. Investigador, Escuela de Educación de la Institución Universitaria Politécnico Grancolombiano. Colombia. Correo electrónico: wperdomo@poligran. $\underline{e d u . c 0}$

2. Magister en Intervención de Sistema Humanos, Universidad Central Colombia. Profesora Escuela de Educación de la Institución Universitaria Politécnico Grancolombiano. Colombia. Correo electrónico: mrortegas@poligran.edu.co 


\title{
Introducción
}

El contexto global actual, caracterizado por una diversidad de problemáticas de orden político, ético, económico, social y cultural, proporciona al escenario pedagógico múltiples desafíos. Estos tienen que ver con la manera en la que se construyen las relaciones, los significados que se gestan, la elección de los contenidos que se enmarcan en los currículos y la diversidad de decisiones de orden ético que retan el quehacer pedagógico.

El cuidado del Otro, el reconocimiento de la diferencia, la constante pregunta sobre las maneras en las que visibilizamos lo que el Otro necesita, desea y sugiere, es el eje central de este artículo. Se enfoca desde una perspectiva del cuidado como modo de ser y como condición inequívoca de que el cuidado no es un acto, sino una actitud. Se intuye la emergente necesidad de reconocimiento de sí mismo y del Otro vital para el cohabitar de las personas, esto conduce hacia una reflexión de la vida misma como un soporte vital de cuidado de sí mismo y del Otro.

Reflexionar sobre el cuidado del Otro y el reconocimiento de la diferencia, nos lleva a entenderlos como elementos relevantes en relación a las dinámicas sociales que se construyen en el contexto educativo. Se trata de un ejercicio ético y reflexivo, que legitima la idea de la consolidación de prácticas y discursos sociales en el marco del espacio pedagógico.

\section{El reconocimiento como elemento vital para el cuidado del Otro}

\author{
Ante todo es necesario cuidar del alma \\ si se quiere que la cabeza y el resto del cuerpo \\ funcionen correctamente
}

Platón

La palabra cuidado relaciona a la persona que cuida como a la persona cuidada; pero cuidar va más allá de una simple atención, de cubrir una necesidad, requiere de un reconocimiento de sí mismo y del Otro, vital para el cohabitar de las personas. Esto, en la premisa que para cuidar necesitamos una conexión verdadera, emocional, que dé importancia al Otro. Es la vida misma, un soporte vital de cuidado del autocuidado, del Otro.

En términos etimológicos la palabra cuidado proviene del latín coera que refiere a aquellas relaciones surgidas del amor o la amistad, la preocupación por el Otro desde el emocionar; esto es así, que requiere de la persona que cuida una atención y comprensión de la necesidad del Otro, aludiendo como Otro, a toda forma de vida o concepción que merece y requiere de nuestra calidad humana, en otras palabras, todo aquello que nos rodea, el mundo en que cohabitamos.

En esta perspectiva, Boff (2002), acuña el término -modo de ser-, esto en la condición inequívoca de que el cuidado no es un acto, sino una actitud, y en esta medida, no es un simple quehacer sino un reconocimiento de todo el sistema como un ser vivo y en el que cada uno es participante como un elemento vital para la sostenibilidad del mismo. En este ser vivo (llamado Gaia), la humanidad al formar parte de este ser vivo, comparte (o debería) procesos de autorregulación y cuidado para su existencia, en esta medida entonces, somos co-responsables de las transformaciones de este ser vivo. El cuidado desde su forma más simple conlleva a la humanidad a su propia sostenibilidad, en palabras de Musil (1988)

Sin duda la suma social de los pequeños esfuerzos diarios, como resultado de su idoneidad para tal suma, trae mucho más energía en el mundo que las obras de los héroes. De hecho, el esfuerzo heroico aparece positivamente, como un grano de arena, en la inmensidad. (p.7) 
Briggs y Peat (1999), señalan en concordancia que es la actitud la que conforma el clima en el que vive el Otro, manifiestan que "si nosotros somos genuinamente felices, positivos, reflexivos, colaboradores y honestos eso influye sutilmente en aquellos que nos rodean" (p.66). Esta influencia sutil que es analizada por ambos autores, lleva a la reflexión acerca de que los actos no constituyen únicamente un efecto para sí mismo, sino que todo acto conlleva a influenciar otros, y en prospectiva la acción debe ser verdadera en sí misma y en su autenticidad, por tanto pasa de ser un acto a ser una actitud.

Por su parte, Noddings (1984) señala que

Cuidar implica salir del propio marco de referencia personal y pasar al del otro. Cuando nos importa, consideramos el punto de vista del otro, sus necesidades objetivas y lo que espera de nosotros. Nuestra atención y nuestra mente están en el cuidado, no en nosotros mismos. Nuestros motivos para actuar, entonces, tienen que ver con los otros, con lo que quiere y con lo que desea. (p.5)

Por consiguiente, el cuidado establece un eje central en la relación con el Otro en la concepción que "el cuidado es esencial para la relación y lo que el cuidador contribuye a la relación es una capacidad de respuesta" (Noddings, 1984, p.181). Donde esta capacidad de respuesta conlleva al afianzamiento de la misma relación desde la igualdad y la equidad, planteado desde la formación del mismo pensamiento ético y moral.

El cuidado entonces forja uno de los principales rasgos de humanidad, en otras palabras, es imprescindible para darnos la condición humana, sin él, estaríamos expuestos a perder una esencia y función vital para el desarrollo de la misma especie. En la misma línea de reconocimiento y cuidado del Otro, la Organización Mundial de la Salud" [OMS] (1946, p.1), define la salud como "un estado de bienestar físico, mental y social, contrario a la ausencia de enfermedad o incapacidad". Así las cosas, el cuidado que señalamos es parte fundamental para el bienestar personal y social, ya que todos los aspectos en la vida de un Ser Humano están correlacionados y de su equilibrio depende el mismo.

Lo anterior, nos lleva a pensar en el cuidado en todas sus dimensiones físicas, emocionales, el reconocimiento del Otro, de sus intereses y necesidades, y en otro aspecto más íntimo, en la búsqueda de la felicidad misma. Echavarría (2009) en éste ámbito de bienestar personal y social, señala una imperiosa necesidad en que:

La formación política y para la ciudadanía orientada por el análisis político contribuye al desarrollo de criterios ético-morales y políticos a partir de los cuales interpelar las responsabilidades que la sociedad civil y las instituciones tienen con los otros ciudadanos. (p. 223)

Es esencial entonces que, en el marco del reconocimiento del Otro, se reconozca también el ejercicio social y ciudadano al que se nos convoca por ser parte de una sociedad, lo que conlleva a considerar el respeto y garantía de derechos y como tal de la reivindicación de una dignidad humana.

En esta condición misma de la especie, el reconocimiento parte de la esencia de los efectos que tienen para algunas personas las actuaciones de otras, ser un objeto social de las acciones de otros (Honnet, 1997). El cuidado cobra nuevamente sentido en este accionar de reconocimiento, que sin el Otro un sujeto no puede incidir sobre las acciones, en el entendido de ser requerida dicha dimensión social como medio intersubjetivo de interacción, es decir a través de la experiencia de reconocerse socialmente. El autor indica que este reconocimiento recíproco, se da de tres formas: el amor, el derecho y la solidaridad.

3. La constitución de la OMS fue adoptada en la Conferencia Sanitaria Internacional celebrada en Nueva York del 19 de junio al 22 de julio de 1946, firmada el 22 de julio de 1946 por los representantes de 61 Estados.

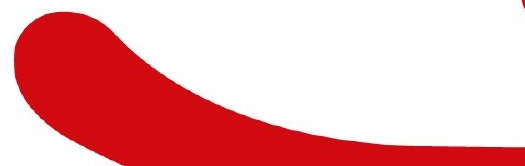


La primera dimensión señalada, el amor, aduce a esa principal fuente de reconocimiento y cuidado, a partir de la relación parental, para luego convertirse en la necesidad primaria de cercanía con el Otro, y a su vez del propio reconocimiento de la afectividad, de lo corporal, de lo espiritual y de la formación de la autoconfianza. En esta misma línea, el amor es un ámbito fundamental de la esencia humana y la negación de su existencia en la convivencia cotidiana sería equivalente a una proporción de sufrimiento en el espacio social que compartimos como seres razonables (Maturana, 1991).

El derecho, como segunda dimensión propuesta por Honneth (1997), nos lleva por una senda de reconocimiento de la individualidad y la libertad personal, pero con todo un despliegue tanto de derechos como de deberes que estructuren a la persona en un ámbito social. Esto permite a afirmar, que la dignidad se alimenta del respeto y auto-respeto, sin el abandono de los deberes personales, sociales o ciudadanos. Para Mead (citado en Honnet, 1997), "en el reconocimiento jurídico el Otro y el ego se respetan recíprocamente como sujetos de derecho, porque en común conocen las reglas sociales, que en su ente comunitario, derechos y deberes se reparten legítimamente" (p.134).

Por último, la solidaridad entendida "como un tipo de relación de interacción en el que los sujetos recíprocamente participan en sus vidas diferenciables, porque se valoran entre sí en forma simétrica" (Honnet, 1997, p.157). Esta forma de reconocimiento reciproco es vital en la mediación para el cuidado del Otro, al descubrir y redescubrir las habilidades propias puestas en beneficio del Otro. Para Araya (2012) citado en Honnet (1997),

Se parte del principio que las personas tienen una interdependencia mutua, la cual es reflejada a través del amor, la amistad y la solidaridad. Esta interacción permite desarrollar la autoconfianza del individuo y además profundizar los valores que se comparten con los Otros y que hacen posible la solidaridad. (p.133)

De este modo, se puede afirmar que el planteamiento del reconocimiento se forja como un eje constructor de nuevas realidades en espacios educativos, la necesidad de las personas de ser reconocidos por los demás grupos, lo cual va más allá del marco jurídico y las libertades de cada ser humano. Los procesos primarios de formación como seres humanos deben constituirse en conocer nuestras propias emociones y explorar a través de esas nuevas sensaciones el reconocimiento del Otro como un ser con los mismos derechos y deberes al descubrir en las acciones del Otro sus emociones y sentimientos.

\title{
La Bioética como un puente posible entre el reconocimiento y el cuidado
}

\author{
La persona bumana conoce y actúa impulsada \\ por resortes no necesariamente racionales, \\ que hacen que la vida bumana tenga un sentido \\ muy profundo y significativo: más natural, \\ relacional, vivencial, creativo, placentero. \\ Se trata de un vivir menos obsesionado por la verdad \\ y más esponjoso delante del misterio de la vida \\ y la felicidad de vivir... \\ Gutiérrez, F. (2006)
}

El término de bioética usado principalmente por el filósofo Fritz Jahr (1927), pero ampliamente expuesto por Van Rensselaer Potter (1971), este último concebía la disciplina como un puente entre dos culturas, que se encuentra interconectado entre las ciencias y las humanidades, pero que se ha visto afectado por el gran distanciamiento que hay entre humanistas, científicos y tecnólogos. En este misma línea, la bioética que describía Potter (citado en Wilches, 2011) expresa que "debía ser una disciplina que partiera y se erigiera sobre lo que él definió como la "crisis de hoy" una crisis generalizada, de claro carácter global que afecta tanto al individuo como a la sociedad y al medio ambiente" (p. 74). 
La crisis a la cual se refiere Potter, parte de la problemática ambiental que afecta a la sociedad en general. Esta crisis exige que la vía con la cual se contrarreste estos efectos adversos debe ser la educación, al partir de una mejor comprensión de la naturaleza humana en su conjunto, su interacción y su desarrollo con el mundo que le rodea; esto se logrará con el gran conocimiento que ha ido adquiriendo el ser humano, haciendo posible la construcción de «puentes hacia el futuro», (Potter, 1971 citado en Wilches, 2011).

Potter, también reclamaba por un nuevo conocimiento para comprendernos como parte de un todo, en el que nuestras acciones y comportamientos representan una cadena de sucesos para la supervivencia humana, en nuestro caso, el transitar por un nuevo paradigma para el buen vivir y la armonía como parte de un ecosistema, que atendiendo a la sugerencia de Gutiérrez y Prieto (2006), vivir menos obsesionado por verdades (apodícticas) y más emocionado por la felicidad misma de vivir.

Bohm (1992) se manifiesta con un primer elemento imprescindible para este cambio necesario en nosotros, la necesidad de un diálogo diferente, de una forma de comunicación no lineal que nos convoque a escucharnos más y a suplir la fuerte necesidad de ser escuchados. Con ello hemos dado cuenta que no queremos más preguntas cerradas con respuestas únicas (o las únicas que quieren ser escuchadas).

Lo anterior, define la necesidad de un cambio a partir de la construcción de nuestra visión sistemática, tal y como lo menciona Capra (1982); que esto nos dirija a un cambio en la forma de pensarnos y del cómo nuestras actuaciones (no solo desde lo científico, sino desde lo espiritual) logran ese entramado necesario para ser parte de una posible transformación social. No se descarta la importancia de esa visión cartesiana, que se ha desarrollado el mundo, sin embargo, es importante reconocer que se debe repensar esa visión, pasar de una visión reductivista a una visión compleja.

Por consiguiente, a partir de todas las experiencias vitales que la humanidad ha afrontado, es imperativo encontrar en el Otro, un interlocutor lleno de ideas y conocimientos, esa es la apuesta de una educación postmoderna: la de conocer y explorar en medio de un debate social los principios y valores que vive el Otro como parte de su existencia. El cuidado solo se puede generar cuando hay un proceso de empatía entre las partes, es decir, la capacidad de ponernos en el lugar del Otro y apropiarnos de la responsabilidad para lograr el bienestar de quienes nos rodean, nos importan o dado el caso así no nos conozcamos; reconocernos en el Otro e intentar contribuir a su cuidado.

Esta posible concepción de alteridad conlleva a su vez, un reto de organización humana en principios y valores universales, al considerar que uno de los principales objetivos que rescatamos es la alusión de las relaciones comportamentales, sus nodos y conexiones, compatibles con el desarrollo de la individualidad y la colectividad, capaces de habitar y co-habitar, al aportar una posible comprensión del sentido de nuestras vidas; quizá conocernos es conocer el ecosistema. La evolución hacia una ciencia que incluya también las relaciones, los sentimientos y las emociones como ejes constructores de una totalidad, permitirá transformar la realidad con un sentido bioético, a partir del reconocimiento y el cuidado del ecosistema.

El reconocimiento entonces, desde una mirada bioética, cobra un rostro, el rostro del Otro y el propio, ambos le ayudan a salir del fangoso terreno de creer únicamente en la individualidad, y convertir el cuidado en el ariete para derribar las murallas de la indiferencia; convocan a una transformación de la conciencia, de las relaciones y de la interacción con los Otros; Levinas (2002) señala que la alteridad "exige para su constitución un sujeto independiente y feliz cuestionado por el hambre del Otro. Un sujeto que sea capaz de sentir vergüenza por la arbitrariedad de su libertad" (p.27). Cuando no existe la emoción, la interacción real tiende a separar el cuidador de lo cuidado.

A través de este reconocimiento y cuidado se potencializa también la salud emocional, esa que es instintiva, que no requiere aprendizaje, que es espontánea, que reconforta el espíritu y el corazón. Si acercamos el reconocimiento y el cuidado del Otro, restituimos en gran parte toda la condición humana personal, social y espiritual. Ahora bien, ese rostro del Otro, no es la suma de unas características corporales, sino que ello contemplaría aspectos

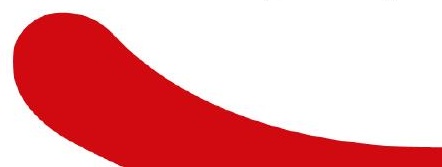


tanto cognitivos como sensitivos (Levinas, 2002), el rostro es totalidad y es infinito (haciendo alusión al título de la obra de este autor).

En la relación rostro-alteridad, se trasciende a una verdadera presencia de un yo hacia un Otro y viceversa (Levinas, 2002); la inmediatez del cara a cara se manifiesta desde la interpelación, que garantiza la primera manifestación de comunicación con el Otro, de dar significado a la corporalidad.

De esta manera, invocar el cuidado en el terreno relacional (ya no desde una mirada individualista) implica reconocer la corporalidad co-construida en las prácticas sociales que asumimos en tales relaciones. Significar las emociones que emergen de una situación común, disponer de la capacidad para ver al Otro en su humanidad y tener la apertura para reflexionar sobre el siguiente paso al reconocimiento, el in situ de cada momento permitirá tomar decisiones que, o bien pueden tener cuidado o por el contrario, pueden detonar en violencia y rechazo.

Sobre el cuerpo vivo que habitamos y sobre el cual se reconoce todo cuidado, tanto físico como emocional, cultural y espiritual, se establecen interrogantes que son pertinentes de enunciar. La pregunta ética sobre el sentir de los cuerpos, el escenario simbólico que es construido sobre la corporalidad, las fuerzas políticas y disciplinantes que lo cubren y lo gestan (sobre todo en el contexto urbano, una relación invisibilizada del Otro en su cuerpo). El Otro no es reconocido en su materia, a excepción que se salga de las categorías hetero-normativas impuestas de belleza, de raza y de género, lo que conlleva al rechazo y pocas veces el cuidado.

De esta manera, el reto ético que dispone esta categoría social, debe ser encarnada en lo cotidiano, en el reconocimiento del Otro, en la diferencia, asumida en la entraña para que los ojos puedan darse la posibilidad afectiva de definitivamente ver al Otro, y que eso sea lo que potencie su capacidad relacional, social y de convivencia.

Maturana (1990) señala la emergencia de únicamente dos emociones que componen éste entramado social: el rechazo y el amor. Al respecto,

El rechazo constituye el espacio de conductas que niegan al otro como legítimo otro en la convivencia; el amor constituye el espacio de conductas que aceptan al otro como un legítimo otro en la convivencia. El rechazo y el amor, sin embargo, no son alternos, porque la ausencia de uno no lleva al otro y ambos tienen como alternativa a la indiferencia. Rechazo y amor, sin embargo, son opuestos en sus consecuencias en el ámbito de la convivencia; el rechazo la niega y el amor la constituyen. El rechazo constituye un espacio de interacciones recurrentes que culmina en la separación; el amor constituye un espacio de interacciones recurrentes que se amplía y puede estabilizarse como tal. Es por esto último que el amor constituye un espacio de interacciones recurrentes en el que se abre un espacio de convivencia donde pueden darse las coordinaciones conductuales de coordinaciones conductuales consensuales que constituyen el lenguaje que funda lo humano, y es por esto que el amor es la emoción fundamental en la historia del linaje homínido a que pertenecemos. (p. 30)

Y es que definitivamente la comprensión del amor siguiendo a Maturana (1990) es "entender que el amor es el punto de partida que configura lo humano, es fundamental, porque nos permite aceptar que lo humano se configura en el vivir y no preexiste" (p.268).

La relación de los conceptos: diálogo, alteridad y amor, conformarían así las bases del puente sobre el cual la bioética, se constituiría en un medio para entender que la persona y personalidad se forjan en el reconocimiento y diálogo con el Otro. 


\title{
El valor educativo del reconocimiento y el cuidado del Otro
}

\author{
Sin aceptación y respeto por sí mismo uno \\ no puede aceptary respetar al otro, y sin aceptar al \\ otro como un legítimo otro en la convivencia, no \\ hay fenómeno social
}

Maturana, H. (1990)

De las anteriores y contundentes perspectivas, renace insistentemente la pregunta: ¿cómo se desarrollan las habilidades amorosas en los escenarios de formación: escuela, familia y universidad? Maturana (1990) expresa que el amor constituye un espacio de interacción recurrente, del cual emergen experiencias de aprendizaje, que marcan el camino, pero, a su vez enceguecen y obstaculizan la capacidad cuidadora. En el ámbito educativo se da la necesidad y la obligación de perpetuar el rol de docentes con el control del poder y del conocimiento.

Por consiguiente, obedecer a estas apuestas en el ambiente educativo conlleva a guiones comportamentales y de significados que promueven lógicas de opresión y poco cuidado. En efecto, es por esta mirada reflexiva que cuestionamos sobre la importancia y énfasis que debe darse sobre el cuidado del Otro, como un valor educativo fundamental, en tiempos donde urge reconocernos unos a Otros para la construcción de una sociedad más justa, equitativa y pacífica.

Existe una emergencia de dar a la pedagogía un carácter más afectivo y emocional, al reconocer que las emociones desempeñan un papel vital en la apertura de los dispositivos de aprendizaje. El reconocimiento del Otro cobra valor educativo cuando se da en contextos sociales, en donde es posible que el escenario de aprendizaje lo permita, las virtudes y capacidades no pueden darse en espacios aislados, el contexto social debe posibilitar el resurgir de dones, virtudes e ideas. En palabras de Flórez (1994) "[...] el hombre se desarrolla, se forma y se humaniza no por un moldeamiento exterior, sino como enriquecimiento que se produce desde el interior mismo del sujeto, como un despliegue libre y expresivo de la propia espiritualidad" (p.108).

Actualmente se reconoce que las metodologías en educación deben ser atrayentes y novedosas, las cuales permitan la construcción colectiva del conocimiento. Por ello, resulta de gran importancia generar -desde la afectividad y el cuidado- espacios dentro del aula que promuevan el reconocimiento del Otro, tanto en la enseñanza como en el aprendizaje. El reconocimiento, la expresión y el cuidado son la respuesta a diferentes estímulos, resultan ser la construcción de un puente que nos lleva a un camino de nuevas adquisiciones.

Desde la perspectiva de Fredrickson y Branigan (2000), las emociones positivas construyen un entramado de recursos con los cuales se logra contrarrestar cualquier aspecto de las emociones negativas, lo que a la vez permite alinear acciones de pensamiento con acciones de hecho de manera positiva. El Ser Humano tiene la responsabilidad social al ser parte del proceso formativo (en todos los escenarios y dimensiones). Ahora bien, la lógica binaria de las emociones se ha ampliado y se ha considerado parte de la responsabilidad que asumimos como docentes, al formar parte de una micro-política y asumir emociones negativas o positivas, que configuran un tipo de disciplinamiento que dejan de potenciar la creatividad y las capacidades del estudiante.

Maturana (1990) reflexiona sobre las emociones y las disposiciones corporales para la acción, es decir, en una emoción cabe un repertorio de acciones que en otra emoción no son posibles; no es lo mismo lo que se ejecuta en la rabia, en la tristeza o en la alegría. De tal modo, que en el cuidado y el reconocimiento del Otro constantemente se debe reflexionar sobre qué tipo de contextos emocionales se co-construyen con los y las aprendientes, relacionadas con las emociones cotidianas como la frustración, el aburrimiento, el miedo, la alegría, la creatividad y la amistad.

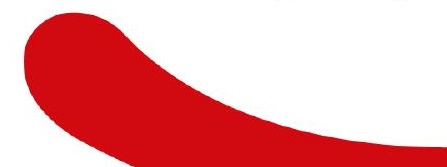


Parte de la tarea que se le adjudica socialmente a los y las docentes dentro de los contextos educativos es identificar y reflexionar sobre el tipo de cuidado y de reconocimiento que se construye desde el actuar dentro y fuera del aula, qué comunica mi cuerpo (la ubicación en el espacio, la disposición del lugar, los gestos, la evaluación y las palabras que se eligen para referirse a los y las estudiantes), qué creencias me impiden o me potencian para poder efectivamente ver y reconocer a cada estudiante, qué puedo hacer para deconstruir aquellas que me imposibilitan escenarios de confianza o aquellas que perpetúan mi rol de poder frente «al que sabe» contra el que «no sabe», qué pasaría sí...

Estas interrogantes de metaconsciencia cotidiana parecen ser infinitas y gestan en su genuina emergencia en la posibilidad de crear escenarios fácticos de cuidado y reconocimiento. Schön (1998) abogaba por un profesional reflexivo, que busque la veracidad de su saber en la acción misma y que ello lo convoque a la autocrítica y evaluación de su conocer y su proceder, y es ésta reflexión en la acción, inherente para comprender el ejercicio docente en la resolución de problemas particulares.

Seguido a esta reflexión, deviene entonces cuestionarnos por las acciones que se desarrollan en el ámbito educativo, en pro de construir escenarios de reconocimiento y cuidado, la base de la educación y el aprendizaje nacen del interés de crear una conciencia responsable, de generar espacios de participación cotidiana activa. Es decir, que la elaboración de modelos educativos se dirija hacia la formación de seres sociales capaces de relacionar conocimientos académicos con sus realidades cotidianas y de esa forma mejorar las condiciones de vida propia y de quienes nos rodean. Esto es una fuerza que potencia las capacidades de los seres humanos en su desarrollo diario, permitiendo la transformación de realidades que mejoran la calidad de vida de quienes forman parte del proceso diario (Maturana, 1991).

La Organización de las Naciones Unidas para la Educación, la Ciencia y la Cultura [UNESCO] ha indicado en repetidas oportunidades la riqueza que trae para una sociedad la comprensión de todas las diferencias individuales, "ver las diferencias individuales no como problemas, sino como oportunidades para enriquecer el aprendizaje" (UNESCO, 2005, p.12). La condición misma del reconocimiento del Otro y sus diferencias promueven el desarrollo personal y la construcción de relaciones entre todos los individuos y el colectivo de una sociedad.

Desde esta perspectiva, la promoción del reconocimiento y el cuidado del Otro en escenarios de aprendizaje no deben limitarse; la acción de ver en el Otro un motivo también de felicidad, encuentro y aprendizaje nos lleva por la senda de la armonía, de la tolerancia y del aceptarnos los unos a los otros. Al considerar ese reconocimiento y cuidado sobre el Otro, y su relación sobre el amor, la relación con el Universo, el cuidado del ambiente, recordamos lo que Swimme (1997) sugiere al respecto,

Para hablar del amor, tenemos que partir por lo que conocemos, por el Universo en constante despliegue en que vivimos. Este ámbito de la existencia es nuestro hogar por antonomasia, el hogar de todos los seres, incluidos los seres humanos. Si queremos aprender algo, tenemos que partir del cosmos, de la Tierra, de las diversas manifestaciones de vida. El encanto, la atracción, es la primera expresión del amor. Piensa en el cosmos, en los cientos de miles de millones de galaxias que se desplazan a toda velocidad por el espacio [...] (p.33)

Comprender la metáfora que significa la educación como ecosistema en nuestros procesos de aprendizaje, significará gratamente comprender las palabras de Morín (1999) al mencionar que "el desarrollo de la aptitud para contextualizar y totalizar los saberes se convierte en un imperativo de la educación" (p.27). El proceso de aprender también nos atrae, ya que como seres humanos siempre buscamos respuestas: cognitivas, sensitivas o espirituales, entre muchas otras.

Así pues, reconocer esta noción ecosistémica de la educación implica identificar prácticas concretas que permitan ser reflexionadas constantemente con el fin de responsabilizarnos con el efecto que tienen en ese todo, del cual somos parte. L. Meza (2012) en su texto «Aprender el cuidado del otro: una urgencia en la formación moral de un país en el cual nos estamos matando» refiere algunas claves que el docente 
necesita en la identificación de prácticas de cuidado con sus estudiantes.

La primera clave es definida como la aceptación del Otro en toda su dimensión, esto en una mirada cultural y social; es el reconocimiento del Otro, de su dignidad personal. Menciona que efectivamente para educar requiere darse al Otro, percibir el mundo del Otro, su contexto, vivir la experiencia juntos y todo esto que lleva a la construcción conjunta.

La segunda clave que menciona el autor es el diálogo, que citando a Noddings (2009) es el eje central en las relaciones, se ejerce el cuidado con el simple hecho de hablar y ser escuchado, en el que cobra gran valor: dar tiempo al Otro y en el que se configuran las coincidencias.

La tercera clave es la práctica y continuidad, aprender a cuidar el acto de ser cuidado, es fundamental que la relación educativa sea consustancial en el reconocimiento y el firme cuidado. Siguiendo a Meza (2012):

[...] para afianzar dicho aprendizaje, debemos procurar que sea duradera. [...] No faltará quien piense que todo esto va a distraer la escuela de su objetivo de formar académicamente a sus estudiantes porque, al final, la sociedad —empezando por sus padres — va a demandarlo. Pues bien, se ha demostrado que las escuelas que aceptan obligaciones de este tipo y brindan servicios en esta clave obtienen mejores rendimientos académicos. Por lo tanto, si queremos que los estudiantes aprendan a recibir cuidado para que con el tiempo desarrollen la capacidad de cuidar a otros, nuestro principal objetivo es conseguir cuidar de ellos. (p.231)

Dicho lo anterior, consideramos que adicional a estas claves que plantea Meza (2012) es necesario que todo el conocimiento construido guarde total y estrecha relación con todo el entorno "una relación inseparable con el medio cultural, social, económico, político y, por supuesto, natural” (Morin, 1999, p.28). Necesitamos pensarnos como ecosistema, como un todo interrelacionado; conectado con el aprendizaje, con el sentir, el ser y el aprender, es de la sorpresa y del asombro que surgen las relaciones (la naturaleza nos lo ha enseñado y lo seguirá haciendo) donde se nutre el encantamiento del aprehender.

\section{Reflexiones finales}

Luego de este recorrido teórico y reflexivo sobre el reconocimiento del Otro, el cuidado, las claves para cuidar al Otro en el contexto pedagógico y otros aspectos, cabe generar algunas reflexiones finales, que más que concluir o cerrar el diálogo, es propiciar la búsqueda de aquellos elementos que se considera claves y que han sido desarrollados en el texto.

Considerar como tarea prioritaria la generación de procesos reflexivos sobre el cuidado en los sistemas humanos y no humanos que componen el contexto educativo, es un ejercicio ético urgente. La importancia de involucrar preguntas dialógicas entre varios agentes que conforman el espacio pedagógico nutre las conversaciones de aperturas creativas hacia modos de convivencia pacíficos en el aula y fuera de él, entre las personas que lo dinamizan, y con todo aquello que nos provee la tierra (Gaia). Esta priorización denota no solo una serie de acciones que den cuenta del cuidado hacia esos sistemas, sino que además se identifica como una actitud constante frente a los significados que construimos en el uso del lenguaje, las acciones y el compromiso no sólo de enfocarnos en generar conocimiento desde los contenidos y las formas, sino sobre todo en la consciencia de revisar procesos, métodos y maneras de cómo nos relacionamos.

Así entonces, la educación como escenario de transformación social tiene como retos la co-construcción de subjetividades cuidadosas y cuidadoras, ciudadanos humanizados con la diversidad y la participación activa y reflexiva de todos los actores que inciden en el proceso educativo de toda sociedad, especialmente aquellos que se inscriben en instituciones como la escuela y/o la universidad. Reconocer la mirada al identificar el cuidado como una actitud nos demanda la producción de espacios de aprendizaje del cuidado (Meza, 2012), que nos inste a deconstruir creencias de la educación tradicional en la forma cómo yo me relaciono y veo al Otro, como un sujeto netamente pensador o productor de conocimiento desde la cognición y el contenido y 
no desde la sensibilidad afectiva del Otro en su humanidad.

En consecuencia con lo anterior, la insistencia de Maturana (1990) en incluir las emociones en el sistema educativo acude a la necesidad urgente de re-pensarnos las maneras en las que construimos relaciones amorosas (reconocimiento del Otro en su legitima diferencia) y en ese sentido a fortalecer las actitudes de cuidado hacia el Otro. Asumir esta actitud cuidadosa exige la consolidación de comunidades despojadas del temor de verse y transformarse en pro de ser éticas en el tipo de relaciones que tejen y con el compromiso firme y consciente de ser gestoras de transformación social.

Cabe resaltar que el abordaje del cuidado y la educación exige más de una mirada y puede ser enriquecida su reflexión si se involucran aspectos desde el enfoque de género, el impacto del cuidado en contextos de violencia política y estructural, la mirada diferencial sobre cómo conciben el cuidado las personas en situación de discapacidad o con diversidad funcional, así como personas de pueblos originarios, afrodescendientes, personas campesinas, entre otros. Dicho esto, sabemos que la pregunta que se abrió en este artículo no es cerrada y que busca provocar otras inquietudes frente a las cuales es fundamental revisar y ampliar, sin embargo, consideramos un ejercicio que reclama nuestra mirada como actores del sistema educativo y en sí, del sistema social.

Finalmente, volcar la mirada hacia los cuerpos que inmersos en un sistema se disciplinan para llevarlos por otra senda la cual es co-crear ejercicios de resistencia frente a dicha acción; todo ello genera preguntas en el cuidado y el respeto por el Otro, la emergencia posible y legítima de la diversidad de los cuerpos con los que compartimos y el que habitamos, sus expresiones, deseos y cuestionamientos. Tenemos la permanente tarea de cuestionarnos sobre un punto final, que cierre cualquier concepción frente al Otro, múltiples cavidades por donde mirarnos e infinidad de caminos por donde aprehender y la gestión de conocer el mundo.

\section{Referencias Bibliográficas}

Araya, J. (2012). Una interpretación del conflicto Mapuhe y el reconocimiento desde la perspectiva de Axel Honneth: una concepción moral y ética. Revista Chilena de Derecho y Ciencia Política, 3(1), pp. 131-146.

Boff, L. (2002). El cuidado esencial. Ética de lo humano, compasión por la tierra. Madrid, España: Editorial Trotta.

Bohm, D. (1992). La totalidad y el orden implicado. Barcelona, España: Editorial Kairós.

Briggs, J. y Peat, D. (1999). Las siete leyes del caos. Las ventajas de una vida caótica. Barcelona, España: Editorial Grijalbo.

Capra, F. (1982). El punto crucial. Ciencia, sociedad y cultura naciente. Buenos Aires, Argentina: Editorial Estaciones.

Echavarría, C. (2009). La formación avanzada en clave ético-moral y política ¿Por qué y para qué la formación política y para la ciudadanía en Colombia? Revista Actualidades Pedagógicas. (54), pp.213225. Bogotá: Universidad de la Salle.

Flórez, R. (1994). Hacia una pedagogía del conocimiento. Santafé de Bogotá, Colombia: Mc Graw Hill.

Fredrickson, B. L. y Branigan, C. (2000). Positive emotions. En Mayne y Bonanno (Eds.), Emotions: current issues and future directions (pp. 123-151). Nueva York, Estados Unidos: Guilford Press. 
Gutiérrez, F. (setiembre de 2006). El sentido de la construcción colectiva del conocimiento. Sendas de Freire. Opresiones, resistencias y emancipaciones en un nuevo paradigma de vida. V Encuentro Internacional de Paulo Freire llevado a cabo con profesionales de la educación en Valencia, España.

Gutiérrez, F. y Prieto, D. (2006). La mediación pedagógica: Apuntes para una educación alternativa. (9a ed.). Buenos Aires, Argentina: Ediciones La Crujía.

Honneth, A. (1997). La lucha por el reconocimiento: por una gramática moral de los conflictos sociales. Barcelona, España: Editorial Crítica.

Jahr, F. (1927). Bio-Ethik. Eine Umschau über die ethischen Beziehungen des Menschen zu Tier und Pflanze. [Bio-Etica: Un análisis de la relación ética de los seres humanos con los animales y las plantas] Kosmos. Handweiser für Naturfreunde, 24(1), pp. 2-4.

Levinas, E. (2002). Totalidad e infinito. Ensayo sobre la exterioridad (6a ed.). Salamanca, España: Ediciones Sígueme.

Maturana, H. (1990). Emociones y lenguaje en educación y política. Santiago, Chile: Ediciones pedagógicas chilenas.

Maturana, H. (1991). El sentido de lo bumano. Santiago, Chile: Ediciones pedagógicas chilenas.

Meza, L. (2012). Aprender el cuidado del otro: una urgencia en la formación moral de un país en el cual nos estamos matando. Revista Actualidades pedagógicas, (60), pp. 215-235.

Morin, E. (1999). La cabeza bien puesta. Repensar la reforma. Reformar el pensamiento. Buenos Aires, Argentina: Nueva Visión.

Musil, R. (1988). El hombre sin atributos (Vol. I). Barcelona, España: Ediciones Seix Barral.

Noddings, N. (1984). Caring. A feminine approach to ethics and moral education. California, Estados Unidos: University of California Press.

Noddings, N. (2009). La educación moral. Propuesta alternativa para la educación del carácter. Buenos Aires, Argentia: Amorrortu.

Organización Mundial de la Salud [OMS]. (1946). Constitución de la Organización Mundial de la Salud. Nueva York: Official Recuperado de World Health Organization. Recuperado de http:/ / www.who.int/governance/eb/ who constitution sp.pdf

Potter, V. (1971). Bioethics: bridge to the future. New York, Estados Unidos: Prentice Hall.

Schön, D. (1998). El profesional reflexivo: cómo piensan los profesionales cuando actúan. Barcelona, España: Paidós.

Swimme, B. (1997). El Universo es un dragón verde. Un relato cósmico de la creación. Santiago, Chile: Sello azul.

UNESCO (2005). Guidelines for inclusion: Ensuring acces to education for all. París: UNESCO. Recuperado de bttp:// unesdoc.unesco.org/images/0014/001402/140224e.pdf

Wilches, Á. (2011). La Propuesta Bioética de Van Rensselaer Potter, cuatro décadas después. Revista Opción, 27 , pp.70-84. 